\title{
Collaborative Edge-Cloud and Edge-Edge Video Analytics
}

\author{
Samaa Gazzaz, Faisal Nawab \\ \{sgazzaz,fnawab\}@ucsc.edu \\ University of California, Santa Cruz
}

\begin{abstract}
According to YouTube statistics [1], more than 400 hours of content is uploaded to its platform every minute. At this rate, it is estimated that it would take more than 70 years of continuous watch time in order to view all content on YouTube, assuming no more content is uploaded. This raises great challenges when attempting to actively process and analyze video content.

Real-time video processing is a critical element that brings forth numerous applications otherwise infeasible due to scalability constraints. Predictive models are commonly used, specifically Neural Networks (NNs), to accelerate processing time when analyzing realtime content. However, applying NNs is computationally expensive. Advanced hardware (e.g. graphics processing units or GPUs) and cloud infrastructure are usually utilized to meet the demand of processing applications. Nevertheless, recent work in the field of edge computing aims to develop systems that relieve the load on the cloud by delegating parts of the job to edge nodes.

Such systems emphasize processing as much as possible within the edge node before delegating the load to the cloud in hopes of reducing the latency. In addition, processing content in the edge promotes the privacy and security of the data. One example is the work by Grulich et al. [2] where the edge node relieves some of the work load off the cloud by splitting, differentiating and compressing the $\mathrm{NN}$ used to analyze the content.
\end{abstract}

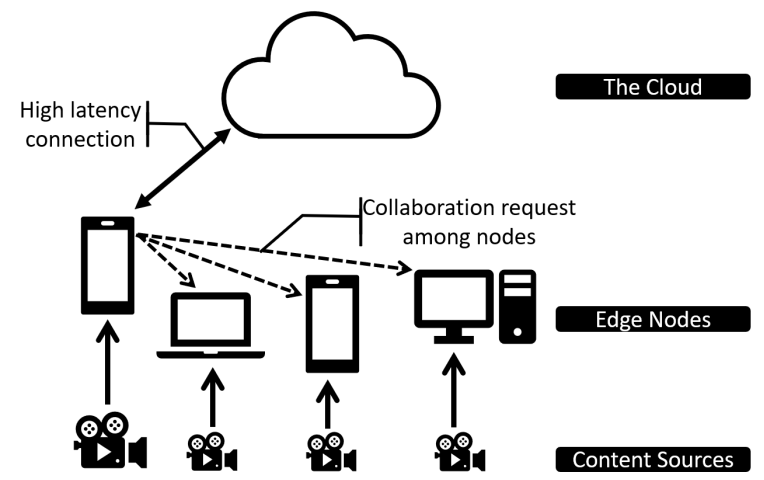

Figure 1: overview of the collaborative approach among edge nodes.

Even though the collaboration between the edge node and the cloud expedites the processing time by relying on the edge node's

Permission to make digital or hard copies of all or part of this work for personal or classroom use is granted without fee provided that copies are not made or distributed for profit or commercial advantage and that copies bear this notice and the full citation on the first page. Copyrights for components of this work owned by others than ACM must be honored. Abstracting with credit is permitted. To copy otherwise, or republish, to post on servers or to redistribute to lists, requires prior specific permission and/or a fee. Request permissions from permissions@acm.org.

SoCC '19, November 20-23, 2019, Santa Cruz, CA, USA

(C) 2019 Association for Computing Machinery.

ACM ISBN 978-1-4503-6973-2/19/11 . .\$15.00

https://doi.org/10.1145/3357223.3366024 capability, there is still room for improvement.

Our proposal aims to utilize the edge nodes even further by allowing the nodes to collaborate among themselves as a para-cloud that minimizes the dependency on the primary processing cloud. We propose a collaborative system solution where a video uploaded on an edge node could be labeled and analyzed collaboratively without the need to utilize cloud resources.

The proposed collaborative system is illustrated in Figure 1. The system consists of multiple edge nodes that acquire video content from different sources. Each node starts the analysis process via a specialized, smaller NN [3] utilizing the edge node's processing power. Whenever the load overwhelms the node or the node is unable to provide accurate analysis via its specialized NN, the node requests other edge nodes to collaborate on the analysis instead of delegating to the cloud resources. This way the high latency is avoided and other edge node processing power is utilized by splitting the NN among the different edge nodes and distributing the processing load between them.

The main contribution of this proposed approach is the alternative conceptualization of collaborative computing: instead of building a system that allows collaboration between edge nodes and the cloud, we explore the prospective of collaboration between edge nodes, minimizing the involvement of the cloud resources even further.

\section{CCS CONCEPTS}

- Information systems $\rightarrow$ Data management systems; • Computing methodologies $\rightarrow$ Distributed computing methodologies.

\section{KEYWORDS}

distributed systems, neural networks, edge computing

ACM Reference Format:

Samaa Gazzaz, Faisal Nawab. 2019. Collaborative Edge-Cloud and EdgeEdge Video Analytics. In ACM Symposium on Cloud Computing (SoCC '19), November 20-23, 2019, Santa Cruz, CA, USA. ACM, New York, NY, USA, 1 page. https://doi.org/10.1145/3357223.3366024

\section{ACKNOWLEDGMENTS}

This research is supported in part by the NSF under grant CNS1815212.

\section{REFERENCES}

[1] 2017. https://fortunelords.com/youtube-statistics/

[2] Grulich, Philipp M., and Faisal Nawab. "Collaborative edge and cloud neural networks for real-time video processing." Proceedings of the VLDB Endowment 11.12 (2018): 2046-2049.

[3] Kang, Daniel, et al. "Noscope: optimizing neural network queries over video at scale." Proceedings of the VLDB Endowment 10.11 (2017): 1586-1597. 